\title{
Transit-Based Emergency Evacuation with Transit Signal Priority in Sudden-Onset Disaster
}

\author{
Ciyun $\operatorname{Lin}^{1,2}$ and Bowen Gong ${ }^{1,2}$ \\ ${ }^{1}$ State Key Laboratory of Automotive Simulation and Control, Jilin University, Changchun 13022, China \\ ${ }^{2}$ Department of Traffic and Transportation, Jilin University, Changchun 13022, China \\ Correspondence should be addressed to Bowen Gong; gongbowen@jlu.edu.cn
}

Received 30 December 2015; Revised 25 March 2016; Accepted 20 April 2016

Academic Editor: Gabriella Bretti

Copyright (c) 2016 C. Lin and B. Gong. This is an open access article distributed under the Creative Commons Attribution License, which permits unrestricted use, distribution, and reproduction in any medium, provided the original work is properly cited.

\begin{abstract}
This study presents methods of transit signal priority without transit-only lanes for a transit-based emergency evacuation in a sudden-onset disaster. Arterial priority signal coordination is optimized when a traffic signal control system provides priority signals for transit vehicles along an evacuation route. Transit signal priority is determined by "transit vehicle arrival time estimation," "queuing vehicle dissipation time estimation," "traffic signal status estimation," "transit signal optimization," and "arterial traffic signal coordination for transit vehicle in evacuation route." It takes advantage of the large capacities of transit vehicles, reduces the evacuation time, and evacuates as many evacuees as possible. The proposed methods were tested on a simulation platform with Paramics V6.0. To evaluate and compare the performance of transit signal priority, three scenarios were simulated in the simulator. The results indicate that the methods of this study can reduce the travel times of transit vehicles along an evacuation route by $13 \%$ and $10 \%$, improve the standard deviation of travel time by $16 \%$ and $46 \%$, and decrease the average person delay at a signalized intersection by $22 \%$ and $17 \%$ when the traffic flow saturation along an evacuation route is $0.8<V / C \leq 1.0$ and $V / C>1.0$, respectively.
\end{abstract}

\section{Introduction}

According to the investigation and statistics of a suddenonset disaster, potential evacuees in personal automobiles are likely to suffer from psychological and physiological shock in a sudden-onset disaster. They are prone to be panic-stricken, have uncontrolled feelings, engage in risk-overtaking, experience vehicle breakdowns, and exhibit abnormal driving psychology and behaviors. A sudden-onset disaster may cause structural damage to traffic infrastructure and functional destruction. These will significantly reduce the passing capacity of a traffic network. In addition, the abnormal driving psychology and behavior of potential evacuees in a sudden-onset disaster often cause traffic accidents and traffic congestion. This situation aggravates the decline in the passing capacity of a traffic network in a sudden-onset disaster. This phenomenon hinders the rapid evacuation of disaster-affected people $[1,2]$.
However, trained transit drivers pay more attention to improving safety awareness and enhancing their ability of vehicle control during an emergency evacuation. In a transitbased evacuation, transit drivers tend to be calmer and are more likely to obey the dissipating commands and optimize evacuation route planning by an emergency operations center. In this case, a transit-based evacuation system can evacuate more disaster-affected people in a timely manner in a sudden-onset disaster. In addition, potential evacuees who do not have access to personal automobiles are more likely to utilize transit, especially buses, to reach safer regions $[3,4]$.

Urban cities in China have fewer cars than any other developed country in the word. Urban areas in China have the characteristics of population concentration and significant travel demand. However, the density of an urban traffic network, the road grade configuration, and the technological level of traffic management is low and unreasonable in China. As a mode of mass transportation, transit has advantages 
such as large capacity, low energy consumption, and high efficiency. Transit transport, especially buses, are considered to be the best approach to solving the problem of emergency evacuation, traffic accidents, and traffic congestion in a sudden-onset disaster. It has a unique role in the evacuation of transit-dependent and vulnerable populations, which are frequently termed special-needs populations, who may lack access to a private vehicle and may need assistance in evacuating $[5,6]$. The importance of transit-based evacuation in China has increased during the last few years.

Transit's capacity to respond to an emergency evacuation is substantially influenced by the traffic infrastructure, advanced transportation management technology, and the socioeconomic and political characteristics of the urban area in which it operates, which affect the size and composition of transit's ridership in a sudden-onset disaster. Transit operates as part of a transportation system in an urban area. Its role in emergency evacuation is dependent on the functioning of this system $[7,8]$. The system needs to increase ridership, decrease evacuation time, enhance transportation system operation efficiency by improving the service quality, and operate reliability and ride comfortability of transit in a suddenonset disaster. Transit signal priority as a favorable treatment of public transport modes within an urban traffic network has a significant contribution to the decline in evacuation time and increase in ridership in a sudden-onset disaster [9].

The importance of transit operations during an emergency evacuation is well established by several evaluation studies; they were conducted due to the recent increase in catastrophic events that required the mass evacuation of a large number of victims $[3,10,11]$. Several studies emphasize the lack of emergency planning for effectively evacuating transit-based populations, especially after experiences such as terrorist attacks, nuclear storage leaks, hurricanes, and earthquakes, which frequently occurred all over the world during the last few decades $[12,13]$. Several studies focus on the role of transit signal priority in relieving traffic congestion and decreasing travel time within an urban traffic network. A literature review revealed that transit signal priority studies focus on the subjects of signal priority strategies [14, 15], signal priority simulation and evaluation [16-18], travel time estimation [11, 19], and transit signal optimization and coordination [20,21]. However, the development of transit signal priority in a sudden-onset disaster for emergency evacuation has not received the same level of attention as the problem of the optimal routing of transit vehicles to maximize the number of people who are evacuated while minimizing evacuation times [22]. Transit signal priority is investigated in the context of daily transit operations with exclusive transit-only lanes. No specific study on this important problem for emergency evacuations has been performed. This study focuses on transit signal priority without transit-only lanes in a sudden-onset disaster to improve transit capacity and reduce transit travel time in a transit-based emergency evacuation with transit signal coordination.

The remainder of the paper is organized as follows. Section 2 explains the level of transit signal priority for an evacuation route and a transit signal priority strategy in a sudden-onset disaster. Section 3 describes the processing of traffic signal arterial coordination for transit signal priority. In Section 4, a simulation and evaluation platform is presented to verify the proposed models of transit signal priority for emergency evacuation without transit-only lanes in a sudden-onset disaster. The results of these various scenarios are discussed in Section 4, and the summary of the findings of this study is concluded in the Section 5.

\section{Transit Signal Priority Level and Strategy for Emergency Evacuation}

The geography and structure of an urban traffic network affect the capacity to evacuate by private vehicle and transit. Geography barriers and functional disruptions of a traffic network impede timely emergency evacuation. Large urban areas with high-capacity and highly-reliable transportation systems generally have the best prospects for successful evacuation. However, capacity bottlenecks at intersections during evacuation routing can reduce the evacuation capacity. Traffic signal coordination with transit signal priority as the favorable treatment for improving traffic capacity and reducing delay within an urban traffic network has a significant contribution to emergency evacuation. It has the ability to regulate and control private vehicles and transit vehicles by traffic signal coordination. Using traffic signal coordination along evacuation route, transit signal priority can significantly reduce delay at an intersection, decrease the evacuation time, and improve the safety, stability, and regularity of evacuation traffic flow.

In a sudden-onset disaster, establishing and maintaining emergency lanes or transit-only lanes along an evacuation route are challenging due to the lack of manpower resources and emergency supplies. Transit signal propriety for emergency evacuation is more practical without emergency lanes or transit-only lanes. In addition, the passengers in transit vehicles include not only typical evacuees, but also sick or injured victims in an emergency evacuation. Therefore, the justification of transit signal priority requires the consideration of the situation of passengers, transit operation statuses, and traffic flow condition along the evacuation route. A traffic signal coordination system can assign transit vehicles different levels of priority and right-of-way to pass through signalized intersections.

The level of priority and right-of-way, a signalized intersection for a transit vehicle, are primarily influenced by transit signal priority strategies. In a traffic signal control system, the strategy for assigning transit signal priority includes a check-in strategy and a checkout strategy. In a check-in strategy, namely, a priority implementation strategy, a traffic signal control system changes a normal traffic signal status to a signal priority status. In a checkout strategy, namely, a signal recovery strategy, a traffic signal control system changes signal priority status to a normal traffic signal status. Priority implementation strategies include green extension, early green, green truncation, phase insertion, phase skipping, and phase rotation. Signal recovery strategies include phase hold, cycle long, cycle short, and cycle smooth [23-25]. 


\section{Transit Signal Priority with Arterial Signal Coordination for Emergency Evacuation}

3.1. Scenarios Hypothesis and Control Logic. This study assumes that no priority demanded transit vehicle leave or enter an approach from side streets. At a road link section along an evacuation route, two sets of sensors are installed: the first set of sensors is installed at the exit of the upstream intersection, and the second set of sensors is installed at the stop-line, namely, the check-in location and the checkout location. These two sets of sensors detect both private vehicles and transit vehicles. The traffic signal control system can detect and provide all information about traffic network flow and the traffic signal controller's status. The transit signal priority control logic is shown in Figure 1.

3.2. Transit Vehicle Arrival Time Estimation. When a transit vehicle arrives and is detected at the check-in location, the traffic signal control system starts to estimate the departure time at which the transit vehicle passes through the signalized intersection along the evacuation route and the arrival time at which the transit vehicle arrives at temporary shelters (fixed points) to pick up evacuees along the evacuation route. Arrive time of traffic vehicle is defined using formula (1). The components of arrive time of traffic vehicle are computed using formulas (2)-(4). The components of arrival time estimation for transit vehicle are shown in Figure 2:

$$
\begin{aligned}
& t_{\mathrm{BUS}}=t_{\text {Dept }}+t_{\text {Cross }}+t_{\text {Arr }}, \\
& t_{\text {Dept }}=\frac{L_{\text {BUS }}}{\bar{v}_{\text {BUS }}}, \\
& t_{\text {Cross }}=\delta \cdot \frac{W}{\bar{v}_{\text {BUS }}}+t_{\text {wait }}, \\
& t_{\text {Arr }}=\frac{L_{\text {Station }}}{\bar{v}_{\text {BUS }}},
\end{aligned}
$$

where $t_{\mathrm{BUS}}$ is the transit vehicle travel time from the transit vehicle check-in location to the next temporary shelter along the evacuation route; $t_{\text {Dept }}$ is the travel time of a transit vehicle from the check-in location to the stop-line of the signalized intersection; $t_{\text {Cross }}$ is the travel time for a transit vehicle to pass through the signalized intersection from the stop-line to the exit of intersection; $t_{\text {Arr }}$ is the travel time from the exit of the intersection to the temporary shelters; $L_{\mathrm{BUS}}$ is the distance from the transit vehicle check-in location to the stop-line of the signalized intersection; $\bar{v}_{\text {BUS }}$ is the average travel speed of transit vehicles; $\delta$ is the intersection width adjustment coefficient; $W$ is the intersection width for a transit vehicle in the approaching direction; $t_{\text {wait }}$ is the waiting time of a transit vehicle that is waiting for a traffic signal to pass through the intersection; and $L_{\text {Station }}$ is the distance from the exit of the intersection to the next temporary shelter.
3.3. Queuing Vehicle Dissipation Time Estimation. In a sudden-onset disaster, establishing and maintaining transitonly lanes for transit-based emergency evacuation are difficult. To provide transit signal priority without transitonly lanes, queuing vehicles should dissipate prior to the signalized intersection in the approaching direction of a transit vehicle. The traffic signal control system estimates the dissipation time of queued vehicles before making a decision regarding how to provide transit signal priority for a transit vehicle. The number of queuing vehicles in the signalized intersection before transit vehicle is defined using formula (5). The dissipation time of queued vehicles is computed using formula (6):

$$
\begin{aligned}
V(t)_{\text {now }} & =V(t)_{\text {up }}-V(t)_{\text {down }}, \\
t_{\mathrm{NQ}} & =\frac{V(t)_{\text {now }}}{S},
\end{aligned}
$$

where $V(t)_{\text {now }}$ is the number of vehicles that queue prior to the signalized intersection in the approaching direction of a transit vehicle; $V(t)_{\text {up }}$ is the number of vehicles detected by the sensors at the check-in location at time $t ; V(t)_{\text {down }}$ is the number of vehicles detected by the sensors at the checkout location at time $t ; t_{\mathrm{NQ}}$ is the queued vehicles dissipation time; and $S$ is the saturation flow rate for vehicles that pass through the signalized intersection.

3.4. Traffic Signal Status Estimation. When a transit vehicle is detected by sensors at the check-in location, the traffic signal control system starts to decide whether and how to provide transit signal priority for transit-based evacuation. The traffic signal status is the main factor for the system to make a decision. The system will estimate the signal status of the transit vehicle in the approaching direction phase when a transit vehicle arrives at the stop-line of the signalized intersection based on the current traffic flow conditions and traffic signal control scheme. The transit vehicle will be confronted with two types of signal statuses when it arrives at the check-in location.

(1) Green Signal Status. When the transit vehicle arrives at the check-in location, the current signal status of the transit vehicle in the approaching direction is a green signal. The signal status when transit vehicle arrives at the check-in location is shown in Figure 3. Before providing traffic signal priority, it needs to estimate whether the transit vehicle can pass through the signalized intersection within the remaining green time. The maximum number of vehicles that can pass through the signalized intersection within the remaining green time is defined using

$$
V(t)_{\text {pass }}=\frac{\left[G(t)_{\text {bus }}-G(t)_{\text {pass }}\right]}{\bar{H}},
$$

where $V(t)_{\text {pass }}$ is the maximum number of vehicles that pass through the signalized intersection within the remaining green time of the transit vehicle in the approaching direction at time $t ; G(t)_{\text {bus }}$ is the phase's green time of the transit vehicle in the approaching direction at time $t ; G(t)_{\text {pass }}$ is the 




FIGURE 1: The control logic of transit signal priority.

passed green time of the transit vehicle in the approaching direction at time $t$; and $\bar{H}$ is the average private car headway from passing through the signalized intersection to the transit approaching direction.

If $V(t)_{\text {now }} \leq V(t)_{\text {pass }}$ and $t_{\text {Dept }} \leq G(t)_{\text {bus }}-G(t)_{\text {pass }}$, the transit vehicle is able to pass through the signalized intersection in the current traffic signal control scheme.

If $V(t)_{\text {now }} \leq V(t)_{\text {pass }}$ and $t_{\text {Dept }}>G(t)_{\text {bus }}-G(t)_{\text {pass }}$, the transit vehicle is not able to pass through the signalized intersection in the current traffic signal control scheme. In the current traffic signal control scheme, the transit vehicle must wait for the next green signal to pass through the signalized intersection. The minimum waiting time is estimated using

$$
t_{\text {wait }}=\left[C-G(t)_{\text {pass }}\right]+t_{\text {Dept }},
$$

where $C$ is the signal cycle of the signalized intersection through which the transit vehicle passes.

If $V(t)_{\text {now }}>V(t)_{\text {pass }}$, the transit vehicle is not able to pass the signalized intersection in the current signal control 


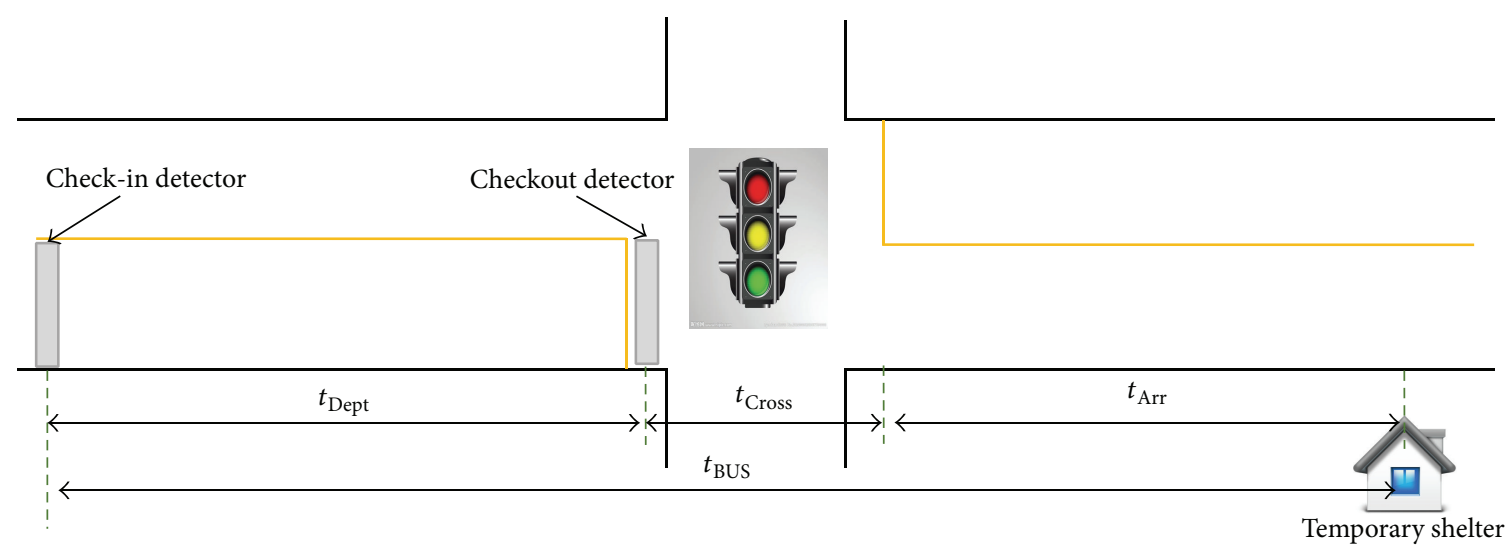

FIGURE 2: The components of arrival time estimation for transit vehicle.

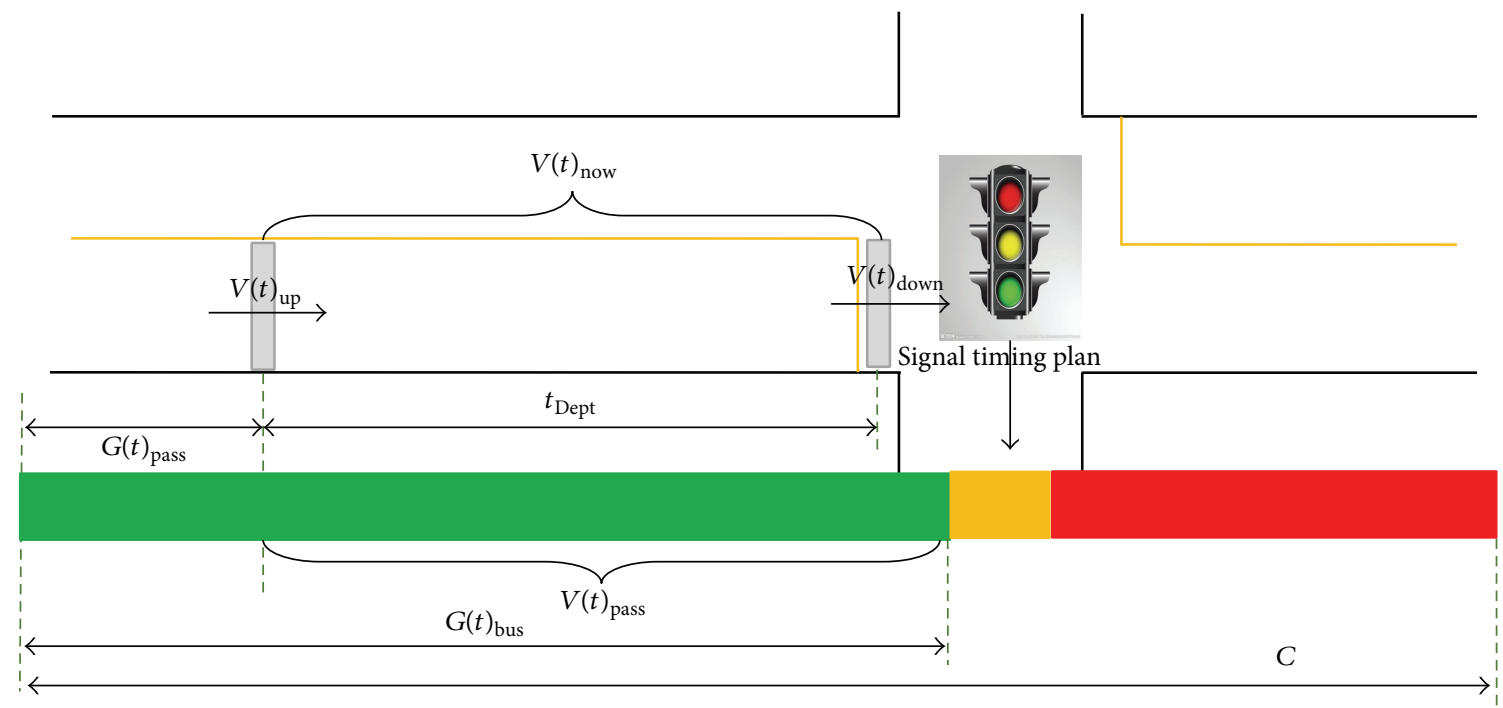

FIGURE 3: The signal status when transit vehicle arrives at the check-in location.

scheme. In the current traffic signal control scheme, the transit vehicle must wait for the next green signal to pass through the signalized intersection. The minimum waiting time is estimated using

$$
t_{\text {wait }}=\left[C-G(t)_{\text {bus }}\right]+\bar{H} \cdot\left[V(t)_{\text {now }}-V(t)_{\text {pass }}\right] .
$$

(2) Red Signal Status. When the transit vehicle arrives at the check-in location, the current signal status of the transit vehicle in the approaching direction is a red signal. The signal status when transit vehicle arrives at the check-in location is shown in Figure 4. Before providing traffic signal priority, it needs to estimate whether the transit vehicle can pass through the signalized intersection within the green phase of the transit vehicle approaching direction. The maximum number of vehicles that can pass through the signalized intersection within the green phase is defined using

$$
V(t)_{\max }=\frac{G(t)_{\text {bus }}}{\bar{H}}
$$

where $V(t)_{\max }$ is the maximum number of vehicles that pass through the signalized intersection to transit vehicle in the approaching direction at time $t$.

If $V(t)_{\text {now }} \leq V(t)_{\max }$ and $t_{\text {Dept }} \geq C-G(t)_{\text {bus }}-$ $R(t)_{\text {pass }}, R(t)_{\text {pass }}$ is the passed red signal time of the transit in the approaching direction at time $t$. The transit vehicle will be faced with the green signal of the transit vehicle in the approaching direction when it arrives at the stopline of the signalized intersection. The transit vehicle will pass through the signalized intersection without giving signal priority strategy.

If $V(t)_{\text {now }} \leq V(t)_{\max }$ and $t_{\text {Dept }}<C-G(t)_{\text {bus }}-R(t)_{\text {pass }}$, the transit vehicle needs to wait for the next green signal to pass through the signalized intersection when the traffic signal control scheme does not change to provide transit signal priority. The waiting time of the transit vehicle at the signalized intersection is defined using

$$
t_{\text {wait }}=C-G(t)_{\text {bus }}-R(t)_{\text {pass }}-t_{\text {Dept }}+t_{\mathrm{NQ}} \text {. }
$$

If $V(t)_{\text {now }}>V(t)_{\max }$, the transit vehicle will queue prior to the signalized intersection approximately two signal cycles 


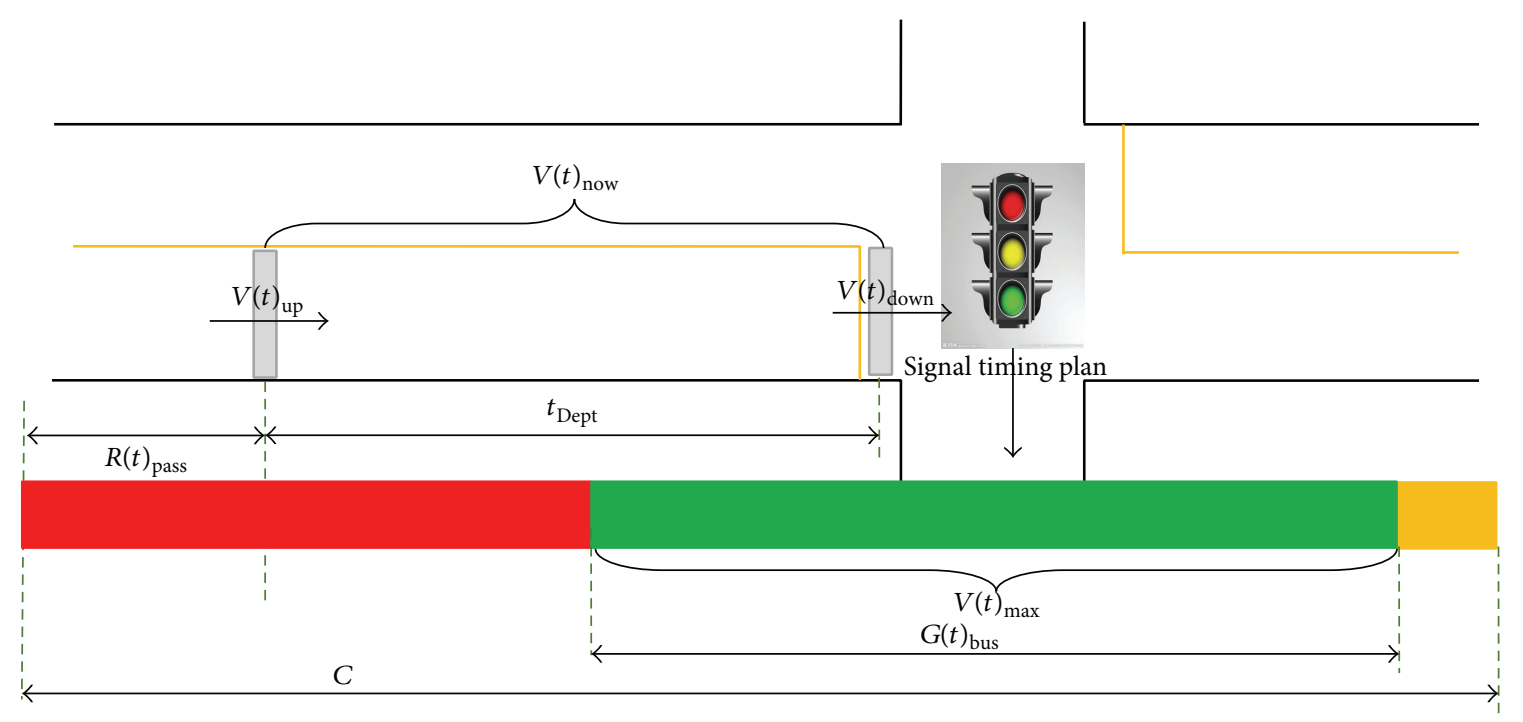

FIgURE 4: The signal status when transit vehicle arrives at the check-in location.

when the traffic signal control scheme does not change to provide transit signal priority. The waiting time of transit vehicle in current signal cycle is $C-R(t)_{\text {pass. }}$. At the end of the current signal cycle, the number of queuing vehicles prior to transit vehicle is $V(t)_{\text {now }}-V(t)_{\max }$. In the next signal cycle, the transit vehicle needs to wait for the green signal to pass through the intersection and the waiting time is $C$ $G(t)_{\text {bus }}$. When the signal turns to green in the next signal cycle, the transit vehicle also needs to wait for the queuing vehicle dispatching the intersection and the waiting time is $\bar{H} \cdot\left(V(t)_{\text {now }}-V(t)_{\max }\right)$. The total waiting time of transit vehicle at the signalized intersection is defined using

$$
\begin{aligned}
t_{\text {wait }}= & 2 C-G(t)_{\text {bus }}-R(t)_{\text {pass }}-t_{\text {Dept }}+\bar{H} \\
& \cdot\left(V(t)_{\text {now }}-V(t)_{\max }\right) .
\end{aligned}
$$

\subsection{Transit Signal Optimization}

3.5.1. Transit Signal Priority. During an emergency evacuation, the most important function of transit vehicles is to transport evacuees when sick or injured victims are not in the transit vehicle. In this situation, the traffic signal control system will consider the arterial traffic capacity and efficiency of the evacuation route. The objective of transit signal optimization is large capacity, safety, and steady travel speeds. The optimization procedure is described as follows.

Step 1. Estimate the current operating status of the transit vehicle. The operating status of the transit vehicle is defined using

$$
\Delta T_{\text {sch }}=T_{\text {Now }}+t_{\text {BUS }}-T_{\text {table }},
$$

where $\Delta T_{\text {sch }}$ is the time difference from which the transit vehicle deviates from the predetermined time when it arrives at the next temporary shelters along the evacuation route; $T_{\text {Now }}$ is the time at which the transit vehicle arrives at the check-in location; $T_{\text {table }}$ is the planning time that the emergency operations center schedules the transit vehicle to arrive at the next temporary shelters along the evacuation route.

If $\left|\Delta T_{\text {sch }}\right| \leq T_{A}$, the transit vehicle is within the normal tolerance range of emergency response dispatching. The traffic signal control system does not need to provide a priority signal. If $\left|\Delta T_{\text {sch }}\right|>T_{A}$, the system proceeds to Step 2. $T_{A}$ is the time difference threshold from which the transit vehicle deviates from the scheduling time that is planned by the emergency operations center.

Step 2. Compute the priority frequency for the transit vehicle to estimate the influence to traffic flow. The signal priority frequency is defined using

$$
\Delta T=T_{\text {Now }}-T_{\text {pre }}
$$

where $\Delta T$ is the time difference between the time transit vehicle arrival at the check-in location and the last time that the traffic signal control system provided a priority signal for the transit vehicle; $T_{\text {pre }}$ is the last time that the traffic signal control system provided a priority signal for the transit vehicle.

If $\Delta T \bmod C<\delta$, transit signal priority may substantially disturb the traffic flow of the disaster-affected area. The traffic signal control system should decline to provide a priority signal for the transit vehicle. If $\Delta T \bmod C \geq \delta$, the system proceeds to Step 3. $\delta$ is the threshold of priority frequency.

Step 3. Traffic signal control system starts to optimize the signal priority strategy for the transit vehicle based on the signal status, transit status, and traffic flow in the network. The traffic signal parameters optimum functions, formulas, and its restrained conditions employ methods and algorithms in the HCM 2000 (Highway Capacity Manual 2000) [26-29], such as determining delay, progression adjustment factor, and signal cycle length. Priority strategy selection and adjustment 
of signal parameters are based on the performance indices of the optimization results from HCM 2000. The traffic signal control system will reevaluate the performance indices of the intersection when the priority strategy is selected. Then, the system decides whether the strategy is suitable for implementing transit signal priority based on the traffic flow conditions of network and the transit operation status. The performance indices of the intersection are computed using

$$
\begin{aligned}
& D_{\text {person }}=\sum_{i \in I}\left(p_{\mathrm{bus}} q_{\mathrm{bus}}^{i}+p_{\mathrm{car}} q_{\mathrm{car}}^{i}\right), \\
& D_{\text {vehicle }}=\sum_{i \in I}\left(q_{\mathrm{bus}}^{i}+q_{\mathrm{car}}^{i}\right),
\end{aligned}
$$

where $D_{\text {person }}$ is the average person delay for a person who is waiting at the signalized intersection during the signal cycle; $p_{\text {bus }}$ and $p_{\text {car }}$ are the average number of riders of the transit vehicle and the private vehicle, respectively; $q_{\text {bus }}^{i}$ and $q_{\text {car }}^{i}$ are the number of transit vehicles and the number of private vehicles, respectively, in the $i$ th inbound direction of the signalized intersection; $D_{\text {vehicle }}$ is the average vehicle delay for a vehicle that is waiting at the signalized intersection during the signal cycle; $D_{\text {person }}^{\prime}$ and $D_{\text {vehicle }}^{\prime}$ are the average person delay and average vehicle delay, respectively, of the signalized intersection prior to justifying the need for priority signal.

If $D_{\text {person }} / D_{\text {person }}^{\prime} \leq \theta_{1}$ and $D_{\text {vehicle }} / D_{\text {vehicle }}^{\prime} \geq \theta_{2}$, then the traffic signal control system will decline to provide a priority signal for the transit vehicle. Otherwise, the system proceeds to Step 4. $\theta_{1}$ and $\theta_{2}$ are the intersection operation efficiency of changing the threshold of average person delay and vehicle delay, respectively.

Step 4. The traffic signal control system evaluates if providing signal priority will disrupt the smooth and steady traffic flow along the evacuation route. We defined formula (16) to evaluate whether priority signal disrupts the smooth and steady traffic flow along the evacuation route:

$$
\varepsilon=\frac{\Delta T_{\mathrm{dev}}}{\Delta G},
$$

where $\varepsilon$ is the influence coefficient of the transit signal priority disrupting the smooth and steady traffic flow along the evacuation route; $\Delta T_{\mathrm{dev}}$ is the green signal time difference of the transit vehicle in the approaching direction phase; $\Delta G$ is the bandwidth of the arterial green wave of the evacuation route.

If $\varepsilon \leq \widetilde{\mathcal{E}}$, the traffic signal control system will provide signal priority for the transit vehicle and use the optimization priority strategy that was optimized by HCM 2000 in Step 3. Otherwise, the traffic signal control system does not change its signal control scheme. $\widetilde{\mathcal{E}}$ is the threshold of the transit signal priority disrupting the smooth and steady traffic flow along the evacuation route.

Step 5. The traffic signal control system starts to recover the signal control scheme when the sensors detect the transit vehicle passing through the signalized intersection at the checkout location. It also needs to optimize the signal parameters among different signal recovery strategies by redesign optimum functions, formulas, and its restrained conditions using the methods and algorithms of the HCM 2000 .

3.5.2. Transit Signal Emergency. In a sudden-onset disaster, an evacuee's psychological and physiological state may suffer different degrees of shock and strike. In a transit-based emergency evacuation, the evacuees in a transit vehicle may include sick or injured victims. They need timely treatment in an emergency shelter in a safer region. In this condition, the traffic signal control system will provide an emergency priority signal for transit vehicles and arterial traffic signal coordination for transit vehicles along an evacuation route. The procedure of providing an emergency priority signal is described as follows.

Step 1. If the phase of the transit vehicle in the approaching direction is green during $t_{\text {Dept }}$, then the traffic signal control system starts arterial traffic signal coordination for the transit vehicle along the evacuation route. Otherwise, the system runs to proceeding Step 2.

Step 2. If the remaining phase green time of the transit vehicle in the approaching direction is less than $t_{\text {Dept }}$ when the transit vehicle arrives at the check-in location, the traffic signal control system will extend the phase green time for the transit vehicle in the approaching direction. The extend time for transit vehicle is defined using formula (17). Otherwise, the system proceeds to Step 3:

$$
t_{\text {extend }}=t_{\text {dept }}-\left[G(t)_{\text {bus }}-G(t)_{\text {pass }}\right] .
$$

Step 3. If the phase of the transit vehicle in the approaching direction is red when the transit vehicle arrives at the checkin location, the next green signal phase services the transit vehicle approaching direction. Then, the traffic signal control system will provide an early phase green starting time of the transit vehicle in the approaching direction. The early time for transit vehicle is defined using

$$
t_{\text {early }}=C-G(t)_{\text {bus }}-R(t)_{\text {pass }}-t_{\text {Dept }} .
$$

Step 4. If the phase of the transit vehicle in the approaching direction is red when the transit vehicle arrives at the checkin location, the next green signal phase is not the phase of the transit vehicle in the approaching direction. The traffic signal control system will optimize the signal parameters among different priority implementation strategies by design optimum functions, formulas, and its restrained conditions using the methods and algorithms in the HCM 2000. The system will evaluate the performance indices and intersection operation efficiency. Then, the traffic signal control system selects the best approach to adjusting the signal parameters to provide emergency signal priority.

Step 5. The traffic signal control system starts to recover the signal control scheme when the sensors detect the transit vehicle passing through the signalized intersection at the 


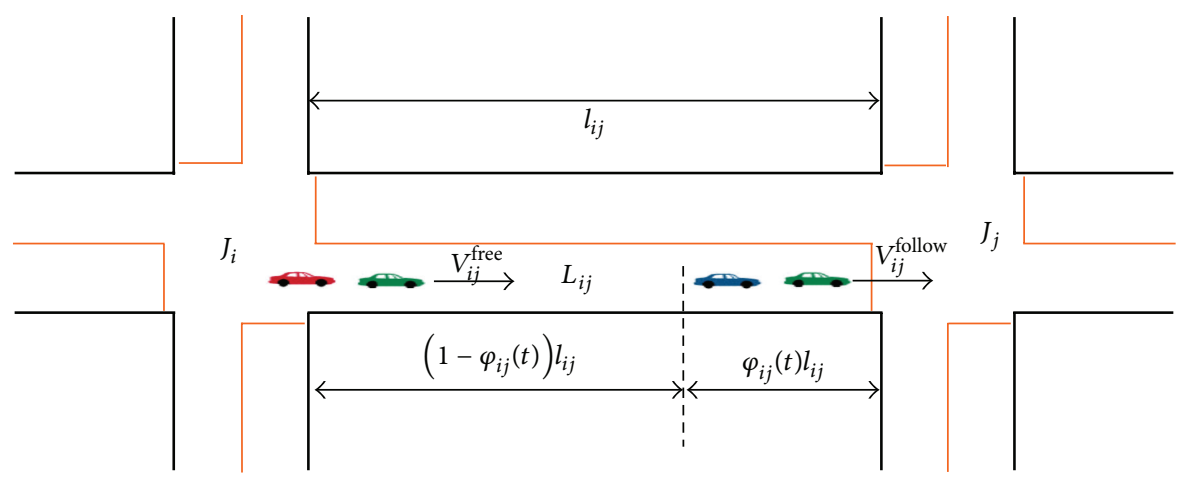

Figure 5: The traffic flow relationship of the adjacent signalized intersection with optimum signal coordination.

checkout location. It also needs to optimize the signal parameters among different signal recovery strategies by redesign optimum functions, formulas, and its restrained conditions using the methods and algorithms in the HCM 2000.

\subsection{Arterial Traffic Signal Coordination for \\ Transit Vehicle along Evacuation Route}

3.6.1. Arterial Signal Offset Optimization. For arterial traffic signal coordination for transit signal priority, the optimum signal coordination ensures that the green signal time and traffic infrastructure are completely utilized by the traffic flow and transit vehicles along the evacuation route. When the last vehicle that is queued at the signalized intersection starts to accelerate and follow the dispatching traffic flow at the $V^{\text {follow }}$ speed, the transit vehicle arrives from the upstream signalized intersection at the $V_{i j}^{\text {free }}$ speed and arrives at the end of the queued vehicle in the downstream intersection. The travel time of transit vehicle at the $V_{i j}^{\text {free }}$ speed in the link section is $\left[1-\varphi_{i j}(t)\right] l_{i j} / V_{i j}^{\text {free }}$. The travel time of transit vehicle at the $V^{\text {follow }}$ speed in the link section is $\varphi_{i j}(t) l_{i j} / V_{i j}^{\text {follow }}$. As to making the transit vehicle arriving follow the dispatching traffic flow at the $V^{\text {follow }}$ speed when it arrives at the end of the queuing vehicle, the signal offset between two adjacent signalized intersections $J_{i}$ (upstream signalized intersection) and $J_{j}$ (downstream signalized intersection) needs to satisfy the following relationship, shown in Figure 5. The signal offset between intersection $J_{i}$ and intersection $J_{j}$ is defined using

$$
O_{i j}(t)+\frac{\varphi_{i j}(t) l_{i j}}{V_{i j}^{\text {follow }}}=\frac{\left[1-\varphi_{i j}(t)\right] l_{i j}}{V_{i j}^{\text {free }}} .
$$

Moving the travel time of transit vehicle at the $V^{\text {follow }}$ speed to the right formula, formula (19) can be rewritten as

$$
O_{i j}(t)=\frac{l_{i j}}{V_{i j}^{\text {free }}}-\left(V_{i j}^{\text {free }}+V_{i j}^{\text {follow }}\right) \cdot \frac{\varphi_{i j}(t) l_{i j}}{\left(V_{i j}^{\text {follow }} \cdot V_{i j}^{\text {free }}\right)},
$$

where $l_{i j}$ is the distance between adjacent intersections $J_{i}$ and $J_{j} ; \varphi_{i j}(t)$ is the density of the link section between $J_{i}$ and $J_{j}$ at time $t ; V_{i j}^{\text {follow }}$ is the vehicle following speed in the link section between $J_{i}$ and $J_{j} ; V_{i j}^{\text {free }}$ is the vehicle free-flow speed in the link section between $J_{i}$ and $J_{j}$ when few vehicles exist in the link section; and $O_{i j}(t)$ is the optimum signal offset between $J_{i}$ and $J_{j}$ at time $t$.

For the bidirectional arterial traffic signal coordination, the signal offset between adjacent intersections $J_{i}$ and $J_{j}$ is defined using

$$
\begin{aligned}
O_{i, j}(t) & =w_{i j} O_{i j}(t)+w_{j i} O_{j i}(t), \\
w_{i j}+w_{j i} & =1,
\end{aligned}
$$

where $O_{i, j}(t)$ is the signal offset of the bidirectional arterial traffic signal coordination between $J_{i}$ and $J_{j}$ at time $t ; O_{j i}(t)$ is the optimum signal offset between $J_{j}$ and $J_{i}$ at time $t$; and $w_{i j}$ and $w_{j i}$ are the signal offset adjustment weight of link section $J_{i}$ to $J_{j}$ and link section $J_{j}$ to $J_{i}$, respectively.

3.6.2. Arterial Signal Offset Online Adjustment. The optimization, adjustment, and maintenance of arterial signal offsets comprises a dynamic process that is based on the changing traffic flow along a traffic arterial. However, arterial signal offsets do not need to be frequently optimized and adjusted, with intervals less than five minutes. If the arterial signal offset is optimized and adjusted at each signal cycle, the optimization objects of traffic signal control performance, which enable smooth and steady traffic flow along an arterial, cannot be acquired. Therefore, the recommended interval for optimizing and adjusting an arterial traffic signal offset is more than five signal cycles. The arterial traffic signal offset adjusting procedure is described as follows.

Step 1. Calculate the interval of the arterial traffic signal offset adjustment time between adjacent intersections. The interval of the arterial traffic signal offset adjustment is defined using

$$
\Delta t_{i, T}^{\mathrm{OS}}=t_{i, T}^{\mathrm{OS}}-t_{i, T-1}^{\mathrm{OS}}
$$

where $\Delta t_{i, T}^{\mathrm{OS}}$ is the interval of the arterial traffic signal offset during signal cycle $T$ of the $i$ th signalized intersection and $t_{i, T}^{\mathrm{OS}}$ and $t_{i, T-1}^{\mathrm{OS}}$ are the signal offset during the signal cycles $T$ and $T-1$, respectively, in the $i$ th signalized intersection. 
Step 2. The system selects the main intersection as the base intersection. The system sets the arterial traffic signal offset adjustment as $\Delta t_{\mathrm{OS}, T}^{\mathrm{key}}$. Therefore, the actual arterial traffic signal offset adjustment time for each intersection is $\Delta t_{\mathrm{OS}, T}^{\mathrm{key}}-$ $\Delta t_{i, T}^{\mathrm{OS}}$.

Step 3. The system employs the temporary signal cycle of each optimum signal cycle for the intersection to replace it with the new common arterial traffic signal cycle. The adjustment increment is set to $f$. Then, the traffic signal control system needs $L=\left(C_{T}-C_{T-1}\right) / f$ times to adjust the arterial signal cycle with the new common arterial signal cycle. $C_{T}$ and $C_{T-1}$ are the common cycles during the signal cycles $T$ and $T-1$, respectively, in the $i$ th signalized intersection.

Step 4. The system selects the maximum arterial signal offset adjustment time for the arterial signal intersection along the evacuation route as $\max \left(\Delta t_{\mathrm{OS}, T}^{\mathrm{key}}-\Delta t_{i, T}^{\mathrm{OS}}\right)$. Then, the system uses $\left[\max \left(\Delta t_{\mathrm{OS}, T}^{\mathrm{key}}-\Delta t_{i, T}^{\mathrm{OS}}\right) / L\right]$ as the increment to adjust the arterial signal offset. The system will execute this procedure until the adjustment time of the arterial signal offset in the signalized intersection is equivalent to $\Delta t_{\mathrm{OS}, T}^{\mathrm{key}}-\Delta t_{i, T}^{\mathrm{OS}}$.

\section{Simulation and Evaluation}

This study employs an urban arterial street in Shanghai city as the evacuation route in the simulation platform. The arterial street is composed of 11 signalized intersections and 11 link sections with eight lanes in bidirection. Among the 11 signalized intersections, four signalized intersections are main road-main road cross intersections and four signalized intersections are main road-minor road cross signalized intersections. The SCATS controls the traffic signals and manages the traffic flow along the urban arterial street. The lengths of the link sections between the signalized intersections are $524.04 \mathrm{~m}, 199.86 \mathrm{~m}, 245.87 \mathrm{~m}, 336.87 \mathrm{~m}, 291.89 \mathrm{~m}, 265.33 \mathrm{~m}$, $173.70 \mathrm{~m}, 206.70 \mathrm{~m}, 143.84 \mathrm{~m}, 617.45 \mathrm{~m}$, and $187.54 \mathrm{~m}$. The snapshot of the evacuation route in the simulation platform is shown in Figure 6.

This study utilizes the software package Paramics V6.0 as the simulation platform. To evaluate and analyze the effect and efficiency of an emergency evacuation with transit signal priority in a sudden-onset disaster, this study develops arterial transit signal priority and coordination methods and algorithms of this paper by $\mathrm{VC}++6.0$ and Paramics API, which include the modules of transit vehicle arrival time estimation, queuing vehicle arrival time estimation, traffic signal status estimation, traffic signal optimization, and arterial traffic signal coordination. By programming with VC++ 6.0 and Paramics API, a plug-in in simulation platform is formed. The traffic flow data of the evacuation route in the simulation platform employs data that were detected by SCATS. Three scenarios are simulated in the simulator to compare the performance indices and evacuation time with different transit signal control logic. The scenarios are described as follows:
(1) Transit-based emergency evacuation is not restricted by traffic signals and does not need to provide arterial signal coordination. The transit vehicle can pass through the signalized intersection without considering the signal status. This scenario is abbreviated as EVP-SCATS.

(2) Transit-based emergency evacuation provides priority signal and arterial signal coordination when the transit vehicle has sick or injured victims. This scenario is abbreviated as EVP-TSAC.

(3) Transit-based emergency evacuation provides priority signal and arterial signal coordination when the transit vehicle has no sick or injured passengers. This scenario is abbreviated as TSP-TSAC.

In the simulation platform, the simulation parameters are as follows: (1) Simulator running time is $3600 \mathrm{~s}$. (2) Simulator's operating step is $2 \mathrm{~s}$. (3) The random number of simulators is six. (4) The departure frequency of the transit vehicle is $15 \mathrm{~min}$. (5) The number of passengers in the transit vehicle is 30 people. (6) Traffic flows were detected by SCATS from August 25, 2014, to August 31, 2014. (7) Signal parameters of the evacuation route in the simulator derive from the original signal plan in SCATS and are generated by the plug-in utilized in the programs VC++ 6.0 and Paramics API to compare the performance of transit signal priority for emergency evacuation in a sudden-onset disaster. (8) Every scenario is simulated three times in the simulation platform.

The simulation results were classified in three groups to analyze the results based on the traffic flow saturation along the evacuation route. The performance results are evaluated and analyzed under the conditions of the traffic flow saturation along the evacuation route: $V / C \leq 0.8,0.8<$ $V / C \leq 1.0$, and $V / C>1.0$, respectively.

(1) Traffic Flow Saturation V/C $\leq 0.8$ along Evacuation Route. The transit vehicle's travel time along the evacuation route is divided into three traffic flow saturations: $V / C \leq 0.5,0.5<$ $V / C \leq 0.7$, and $V / C \leq 0.8$. In the three simulations, this study selected 20 sequence samples of the best performance simulation results to compare. The emergency evacuation times of the transit vehicle in different traffic signal control scenarios are shown in Figure 7.

For the traffic flow saturation $V / C \leq 0.5$ along the evacuation route, the traffic volume is small and the queue lengths at the signalized intersections are short. In this situation, the traffic flow has a minimal effect on the transit vehicle in an emergency evacuation, and the transit vehicle encounters minimal delay at the signalized intersections. The travel times of the transit vehicle along the evacuation route do not significantly differ under different traffic signal control scenarios. As the traffic flow saturation along the evacuation route increases, the travel time of the transit vehicle along the evacuation route is gradually affected by the traffic flow. The travel times of transit vehicles along the evacuation route do not significantly differ in different traffic signal control scenarios when traffic flow saturation along the evacuation route falls between 0.5 and 0.7. When the traffic flow saturation along the evacuation route falls 


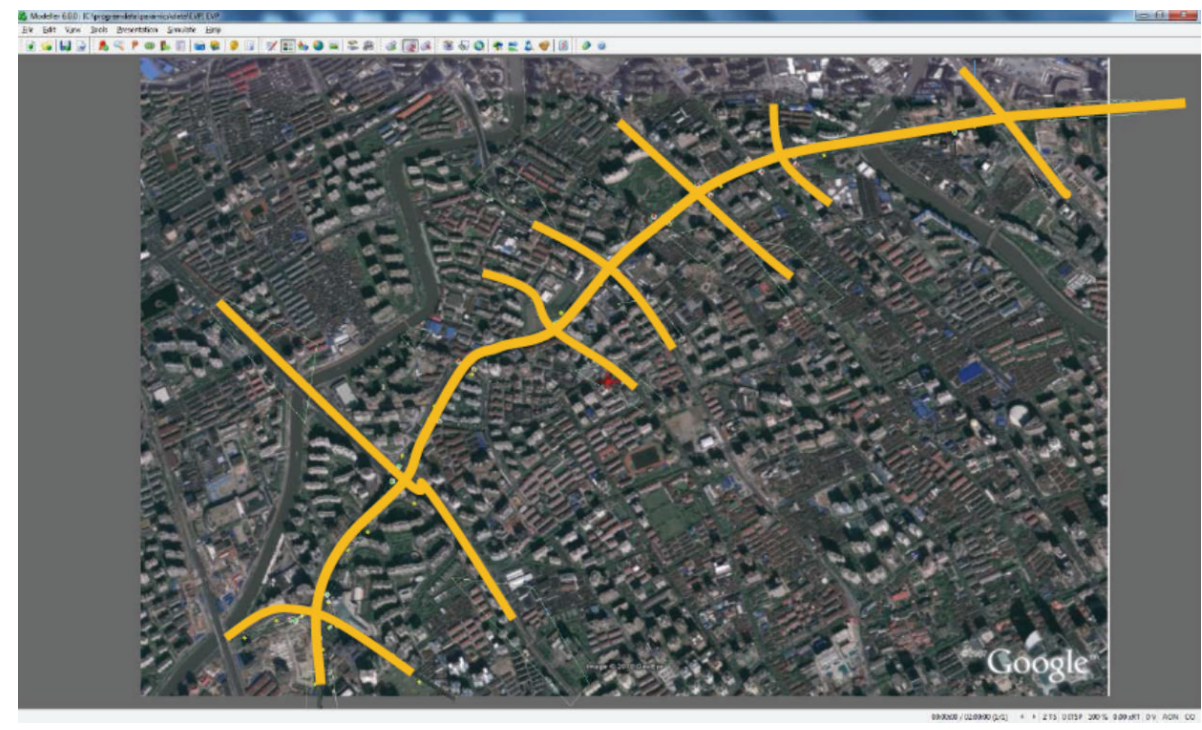

FIGURE 6: The snapshot of evacuation route.

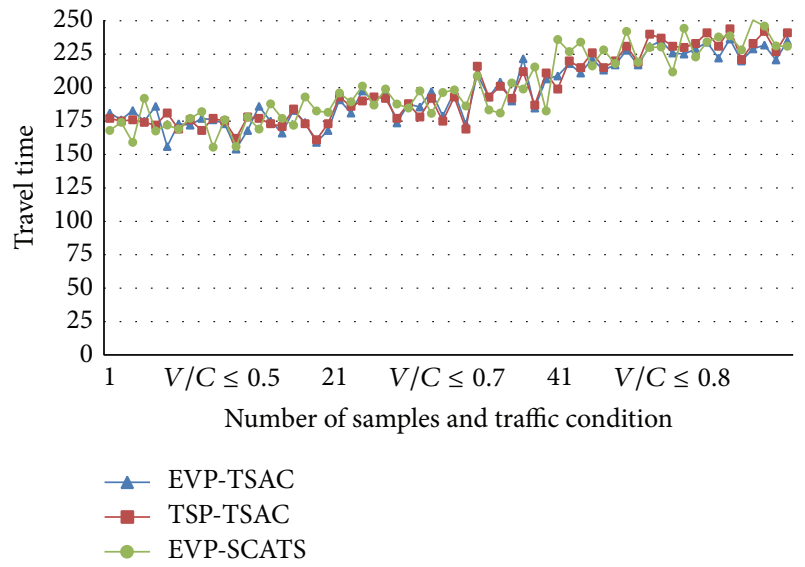

Figure 7: Transit travel time with $V / C \leq 0.8$ for different traffic signal control scenarios.

between 0.7 and 0.8 , the average travel time of the transit vehicle and the standard deviation of the travel time along the evacuation route are $200.41 \mathrm{~s}$ and $26.17 \mathrm{~s}$, respectively, for the traffic signal control scenario of EVP-SCATS. The average travel time of the transit vehicle and the standard deviation of the travel time along the evacuation route are $198.14 \mathrm{~s}$ and $23.71 \mathrm{~s}$, respectively, for the traffic signal control scenario of EVP-TSAC. The average travel time of the transit vehicle and the standard deviation of the travel time along the evacuation route are $198.43 \mathrm{~s}$ and $25.36 \mathrm{~s}$, respectively, for the traffic signal control scenario of TSP-TSAC.

The performance indices of the transit vehicle and the private vehicle in the signalized intersection for different traffic flow saturations and traffic signal control scenarios are listed in Table 1.

As shown in Table 1, the travel time of the transit vehicle and the delay of private vehicle increase as the

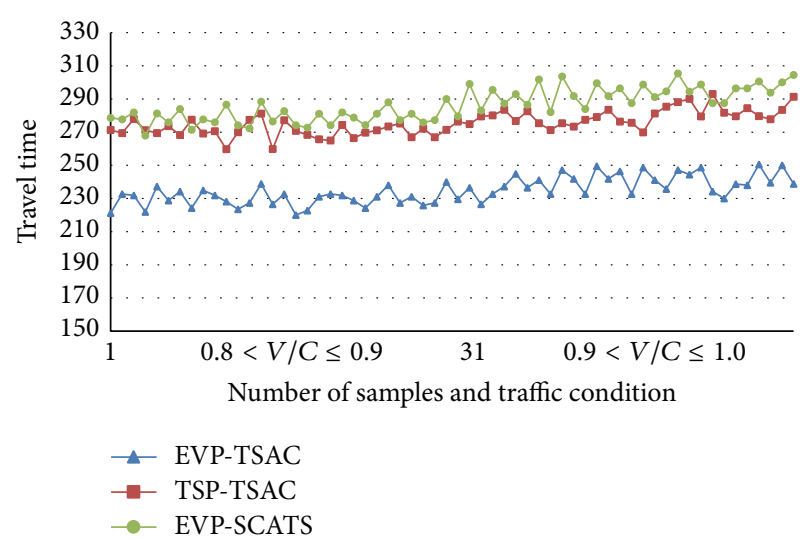

FIgure 8: Transit travel time with $0.8<V / C \leq 1.0$ for different traffic signal control scenarios.

traffic flow saturation increases. At low traffic flow saturation along the evacuation route, the traffic signal control system demonstrates that transit signal priority does not significantly influence other vehicles along the evacuation route and along the side street of the evacuation route.

(2) Traffic Flow Saturation $0.8<V / C \leq 1.0$ along the Evacuation Route. The travel time of the transit vehicle along the evacuation route is divided into two traffic flow saturations: $0.8<V / C \leq 0.9$ and $0.9<V / C \leq 1.0$. In the three simulations, this study selects 30 sequence samples of the best performance simulation results for comparison. The emergency evacuation time of a transit vehicle in different traffic signal control scenarios are shown in Figure 8.

The travel time of a transit vehicle can be effectively reduced by executing transit signal priority and priority signal arterial coordination when a transit vehicle has sick and injured victims. However, as the traffic flow saturation increases along the evacuation route, the traffic flow impacts 
TABLE 1: The performance indices of transit vehicle and private vehicle at $V / C \leq 0.8$.

\begin{tabular}{lccccccc}
\hline \multirow{2}{*}{ Traffic condition } & \multirow{2}{*}{ Control logic } & \multicolumn{2}{c}{ Transit vehicle } & \multicolumn{2}{c}{ Private vehicle } & \multicolumn{2}{c}{ Whole intersection } \\
& & TT & STDEV & Delay-M & Delay-C & V-delay & P-delay \\
\hline \multirow{3}{*}{$V / C \leq 0.5$} & EVP-SCATS & 199.25 & 20.73 & 4.54 & 7.79 & 6.38 & 5.84 \\
& TSP-TSAC & 198.72 & 19.64 & 4.16 & 8.26 & 6.46 & 5.72 \\
& CC & -0.27 & -5.26 & -8.37 & 6.03 & 1.25 & -2.05 \\
\hline \multirow{3}{*}{$V / C \leq 0.7$} & EVP-SCATS & 210.42 & 19.74 & 10.19 & 13.59 & 12.69 & 12.07 \\
& TSP-TSAC & 208.69 & 19.18 & 9.87 & 14.27 & 12.87 & 1.32 \\
& CC & -0.82 & -2.84 & -3.14 & 5.00 & 1.42 & -6.21 \\
\hline \multirow{3}{*}{$V / C \leq 0.8$} & EVP-SCATS & 232.37 & 20.37 & 10.87 & 23.32 & 17.00 & 16.43 \\
& TSP-TSAC & 226.89 & 19.14 & 10.23 & 22.60 & 17.12 & 16.05 \\
& CC & -2.36 & -6.04 & -5.89 & -3.09 & 0.71 & -2.31 \\
\hline
\end{tabular}

Note: TT: travel time of transit vehicle along evacuation route (s). STDEV: standard deviation of travel time (s). Delay-M: average delay of private vehicle in the signalized intersection along the evacuation route (s). Delay-C: average delay of private vehicle on the side street along the evacuation route (s). V-delay: average vehicle delay in the signalized intersection (s); P-delay: average person delay in the signalized intersection $(\mathrm{s})$. CC $=($ TSP-TSAC - TSP-SCATS $) *$ 100/TSP-SCATS (\%).

TABLE 2: The performance indices of transit vehicle and private vehicle at $0.8<V / C \leq 1.0$.

\begin{tabular}{lccccccc}
\hline \multirow{2}{*}{ Traffic condition } & \multirow{2}{*}{ Control logic } & \multicolumn{2}{c}{ Transit vehicle } & \multicolumn{2}{c}{ Private vehicle } & \multicolumn{2}{c}{ Whole intersection } \\
& & TT & STDEV & Delay-M & Delay-C & V-delay & P-delay \\
\hline \multirow{3}{*}{$V / C \leq 0.9$} & EVP-SCATS & 282.72 & 10.73 & 4.54 & 7.79 & 6.38 & 5.84 \\
& TSP-TSAC & 254.25 & 9.64 & 4.16 & 8.26 & 6.46 & 4.72 \\
& CC & -10.07 & -10.16 & -8.37 & 6.03 & 1.25 & -19.18 \\
\hline \multirow{3}{*}{$V / C \leq 1.0$} & EVP-SCATS & 316.86 & 9.74 & 10.19 & 13.59 & 12.69 & 12.07 \\
& TSP-TSAC & 272.86 & 8.18 & 9.87 & 14.27 & 12.87 & 9.32 \\
& CC & -13.89 & -16.02 & -3.14 & 5.00 & 1.42 & -22.78 \\
\hline
\end{tabular}

on transit vehicles becoming significant. In addition, the travel time of the transit vehicle along the evacuation route increases as the traffic flow saturation increases. When the traffic flow saturation along evacuation route falls between 0.8 and 1.0, the average travel time of the transit vehicle and the standard deviation of the travel time of along the evacuation route are is $286.20 \mathrm{~s}$ and $9.77 \mathrm{~s}$, respectively, for the traffic signal control scenario of EVP-SCATS. The average travel time of the transit vehicle and the standard deviation of travel time along the evacuation route are $234.72 \mathrm{~s}$ and $7.98 \mathrm{~s}$, respectively, for the traffic signal control scenario of EVP-TSAC. The average travel time of the transit vehicle and the standard deviation of travel time along the evacuation route are $275.46 \mathrm{~s}$ and $7.12 \mathrm{~s}$, respectively, for the traffic signal control scenario of TSP-TSAC.

The performance indices of the transit vehicle and the private vehicle in the signalized intersection for different traffic flow saturations and traffic signal control scenarios are listed in Table 2.

(3) Traffic Flow Saturation $V / C>1.0$ along Evacuation Route. The traffic flow saturation $V / C>1.0$ along evacuation route, which occurs during the peak-hour in the disasteraffected regions experiences a significant traffic demand to evacuate. In this situation, the evacuation route may experience traffic congestion or traffic jam. Because the queue lengths of vehicles in signalized intersections are long, and transit vehicles have difficulty passing through signalized

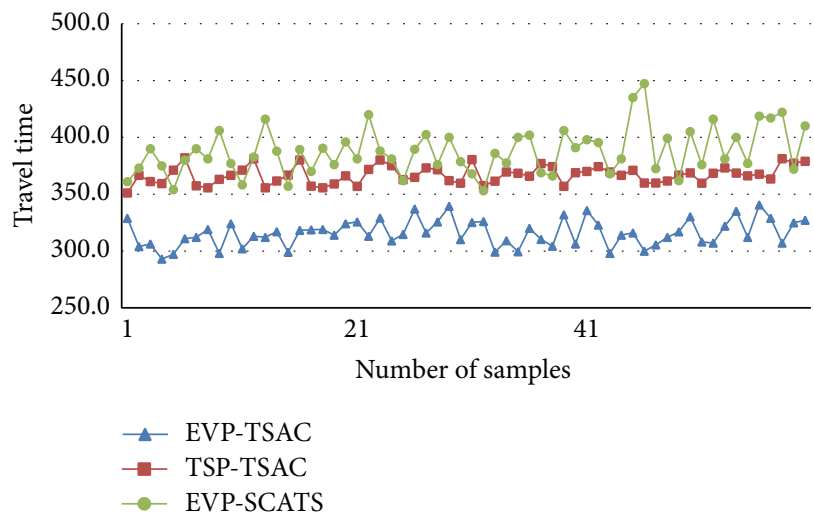

Figure 9: Transit travel time with $V / C>1.0$ for different traffic signal control scenarios.

intersections without the transit-only lanes. In the three simulations, this study selects 60 sequence samples of the best performance simulation results for a comparison. The emergency evacuation times of the transit vehicle in different traffic signal control scenarios are shown in Figure 9.

For the traffic flow saturation of $V / C>1.0$ during an evacuation, the average travel time of the transit vehicle and the standard deviation of the travel time along the evacuation route are $387.69 \mathrm{~s}$ and $20.28 \mathrm{~s}$, respectively, for the traffic signal control scenario of EVP-SCATS. The average travel 
TABLE 3: The performance indices of the transit vehicle and the private vehicle at $V / C>1.0$.

\begin{tabular}{lccccccc}
\hline \multirow{2}{*}{ Traffic condition } & \multirow{2}{*}{ Control logic } & \multicolumn{2}{c}{ Transit vehicle } & \multicolumn{2}{c}{ Private car } & \multicolumn{2}{c}{ Whole intersection } \\
& & TT & STDEV & Delay-M & Delay-C & V-delay & P-delay \\
\hline \multirow{3}{*}{$V / C>1.0$} & EVP-SCATS & 396.69 & 20.28 & 19.60 & 28.46 & 26.96 & 24.80 \\
& TSP-TSAC & 357.02 & 10.75 & 16.90 & 31.69 & 24.65 & 20.40 \\
& CC & -10.00 & -46.99 & -13.78 & 11.35 & -8.57 & -17.74 \\
\hline
\end{tabular}

time of the transit vehicle and the standard deviation of the travel time along the evacuation route are $315.77 \mathrm{~s}$ and $11.69 \mathrm{~s}$, respectively, for the traffic signal control scenario of EVPTSAC. The average travel time of the transit vehicle and the standard deviation of the travel time along the evacuation route are $367.02 \mathrm{~s}$ and $7.75 \mathrm{~s}$, respectively, for the traffic signal control scenario of TSP-TSAC.

The performance indices of the transit vehicle and the private vehicle in the signalized intersection for different traffic signal control scenarios with traffic flow saturations $V / C>1.0$ are listed in Table 3.

\section{Conclusions}

This study proposes methods of transit signal priority without transit-only lanes for transit-based emergency evacuations in a sudden-onset disaster. The motivations to propose the method in this study are the following:

(1) In a sudden-onset disaster, establishing and maintaining emergency lanes or transit-only lanes along evacuation route are difficult due to lacking of manpower resources and emergency supplies. Transit signal propriety for emergency evacuation is more practical without emergency lanes or transit-only lanes.

(2) A transit-based emergency evacuation can take advantage of the large capacity, high efficiency, and low energy of transit vehicles. In a transit-based evacuation, transit drivers tend to be calmer and more likely to obey dissipating commands from and optimize evacuation route planning by an emergency operations center. In this case, a transit-based evacuation system can evacuate a greater number of disasteraffected people in a timely manner in a sudden-onset disaster.

(3) Transit signal priority can significantly reduce the evacuation time and improve the transit capacity for an emergency evacuation in a sudden-onset disaster. In addition, the passengers in transit vehicles include not only typical evacuees, but also sick or injured victims in an emergency evacuation. Therefore, a traffic signal control system can provide different signal priority strategies that consider the situations of passengers, transit operation statuses, and traffic flow conditions along an evacuation route.

The transit signal priority control logic of this paper includes five sections: "transit vehicle arrival time estimation," "queuing vehicle dissipation time estimation," "traffic signal status estimation," "transit signal optimization," and "arterial traffic signal coordination for transit vehicle in evacuation route." The signal attempts to reduce the evacuation time for transit vehicles along an evacuation route with minimal delay in signalized intersection to justify the merit of transit signal priority and arterial priority signal coordination.

This study employs a simulation platform with Paramics V6.0 and develops a plug-in simulation platform by $\mathrm{VC}++$ and Paramics API. Thus, the simulation platform can simulate and evaluate the performance effect and efficiency of the transit signal priority method and the algorithm proposed in this paper. The simulator utilizes an urban main road in Shanghai city as the evacuation route in the simulation. The traffic data input in the simulator is detected by SCATS, which controls and manages the urban main road in Shanghai from August 25, 2014, to August 31, 2014. To evaluate and compare the performance of transit signal priority, three scenarios are simulated in the simulator: EVP-SCATS, EVP-TSAC, and TSP-TSAC.

The simulation results indicate that when the traffic flow saturation along the evacuation route is $V / C \leq 0.8$, the travel times of the transit vehicles for different traffic signal control scenarios do not differ significantly. When the traffic flow saturation along the evacuation route falls between 0.8 and 1.0, the travel time of the transit vehicle decreases by $13 \%$, the standard deviation of the travel time increases by $16 \%$, and the average person delay in signalized intersection decreases by $22 \%$. When the traffic flow saturation along the evacuation route $V / C>1.0$, the travel time of the transit vehicle decreases by $10 \%$, the standard deviation of the travel time increases by $46 \%$, and the average person delay in the signalized intersection decreases by $17 \%$. The traffic signal control scenarios of EVP-SCATS, EVP-TSAC, and TSPTSAC, which are discussed in this paper, can significantly reduce the travel times of a transit vehicle, improve the standard deviation of the travel time, and decrease the average person delay in a signalized intersection along an evacuation route when the traffic flow saturation $V / C>0.8$.

\section{Competing Interests}

The authors declare that there are no competing interests regarding the publication of this paper.

\section{Acknowledgments}

The authors acknowledge that National Natural Science Foundation of China (Grant no. 51408257 and no. 
51308248), Youth Scientific Research Fund of Jilin (Grant no. 20140520134JH), and Specialized Research Fund for the Doctoral Program of Higher Education of China (Grant no. 20120061120046) partly support this work.

\section{References}

[1] S. A. Parr, E. I. Kaisar, and A. Stevanovic, "Application of transit signal priority for no-notice urban evacuation," Natural Hazards Review, vol. 15, no. 2, pp. 167-170, 2014.

[2] S. Sharma, "Simulation and modeling of group behavior during emergency evacuation," in Proceedings of the IEEE Symposium on Intelligent Agents (IA '09), pp. 122-127, April 2009.

[3] E. Morgul, O. Cavus, K. Ozbay, and C. Iyigun, "Modeling of bus transit driver availability for effective emergency evacuation in disaster relief," Transportation Research Record, no. 2376, pp. 45-55, 2013.

[4] H. Naghawi and B. Wolshon, "Operation of multimodal transport system during mass evacuations," Canadian Journal of Civil Engineering, vol. 42, no. 2, pp. 81-88, 2015.

[5] D. L. Miller, A. K. Kanafani, and O. E. Skinner, The Role of Transit in Emergency Evacuation: Committee on the Role of Public Transportation in Emergency Evacuation, Transportation Research Board, Washington, DC, USA, 2008.

[6] F. Sayyady and S. D. Eksioglu, "Optimizing the use of public transit system during no-notice evacuation of urban areas," Computers and Industrial Engineering, vol. 59, no. 4, pp. 488495, 2010.

[7] N. Elmitiny, S. Ramasamy, and E. Radwan, "Emergency evacuation planning and preparedness of transit facilities traffic simulation modeling," Transportation Research Record, no. 1992, pp. 121-126, 2007.

[8] H. Naghawi and B. Wolshon, "Performance of traffic networks during multimodal evacuations: simulation-based assessment," Natural Hazards Review, vol. 13, no. 3, pp. 196-204, 2012.

[9] C. Diakaki, M. Papageorgiou, V. Dinopoulou, I. Papamichail, and M. Garyfalia, "State-of-the-art and -practice review of public transport priority strategies," IET Intelligent Transport Systems, vol. 9, no. 4, pp. 391-406, 2015.

[10] A. Alam and M. Hatzopoulou, "Reducing transit bus emissions: alternative fuels or traffic operations?" Atmospheric Environment, vol. 89, pp. 129-139, 2014.

[11] L. Henson and A. Lyons, "Integrating bluetooth-based travel time data for real-time traffic operations use," Ite JournalInstitute of Transportation Engineers, vol. 84, no. 2, pp. 46-47, 2014.

[12] H. Naghawi and B. Wolshon, "Transit-based emergency evacuation simulation modeling," Journal of Transportation Safety \& Security, vol. 2, no. 2, pp. 184-201, 2010.

[13] T. Litman, "Lessons from Katrina and Rita: what major disasters can teach transportation planners," Journal of Transportation Engineering, vol. 132, no. 1, pp. 11-18, 2006.

[14] Y. J. Lin, X. F. Yang, G.-L. Chang, and N. Zou, "Transit priority strategies for multiple routes under headway-based operations," Transportation Research Record, vol. 2356, pp. 34-43, 2013.

[15] L. Wang and Y. Bie, "An adaptive model for calculating the correlation degree of multiple adjacent signalized intersections," Mathematical Problems in Engineering, vol. 2013, Article ID 247184, 13 pages, 2013.

[16] M. Shourijeh, A. Faghri, and A. Stevanovic, "Simulationbased planning framework for choosing where to implement bus signal priority in mixed-mode operations," Transportation Research Record, no. 2352, pp. 31-40, 2013.

[17] M. S. Ghanim, F. Dion, and G. Abu-Lebdeh, "The impact of dwell time variability on transit signal priority performance," Canadian Journal of Civil Engineering, vol. 41, no. 2, pp. 154-163, 2014.

[18] S. I. Guler and M. Menendez, "Analytical formulation and empirical evaluation of pre-signals for bus priority," Transportation Research Part B: Methodological, vol. 64, pp. 41-53, 2014.

[19] W. J. Ma, K. L. Head, and Y. H. Feng, "Integrated optimization of transit priority operation at isolated intersections: a person-capacity-based approach," Transportation Research Part C: Emerging Technologies, vol. 40, pp. 49-62, 2014.

[20] Q. He, K. L. Head, and J. Ding, "Multi-modal traffic signal control with priority, signal actuation and coordination," Transportation Research Part C: Emerging Technologies, vol. 46, pp. 65-82, 2014.

[21] X. S. Zeng, Y. L. Zhang, K. N. Balke, and K. Yin, "A real-time transit signal priority control model considering stochastic bus arrival time," IEEE Transactions on Intelligent Transportation Systems, vol. 15, no. 4, pp. 1657-1666, 2014.

[22] C.-Y. Lin, B.-W. Gong, D.-X. Zhao, and Z.-S. Yang, “Timing optimization of traffic signals for emergency evacuation in sudden-onset disasters," Journal of South China University of Technology (Natural Science Edition), vol. 41, no. 2, pp. 58-65, 2013.

[23] A. Rodriguez and A. R. Danaher, "Operational comparison of transit signal priority strategies," Transportation Research Record, vol. 2418, pp. 84-91, 2014.

[24] Y. Lin, X. Yang, N. Zou, and M. Franz, “Transit signal priority control at signalized intersections: a comprehensive review," Transportation Letters, vol. 7, no. 3, pp. 168-180, 2015.

[25] O. J. Ibarra-Rojas, F. Delgado, R. Giesen, and J. C. Muñoz, "Planning, operation, and control of bus transport systems: a literature review," Transportation Research Part B: Methodological, vol. 77, pp. 38-75, 2015.

[26] S. Göttlich, M. Herty, and U. Ziegler, "Modeling and optimizing traffic light settings in road networks," Computers \& Operations Research, vol. 55, pp. 36-51, 2015.

[27] R. Liu and M. Smith, "Route choice and traffic signal control: a study of the stability and instability of a new dynamical model of route choice and traffic signal control," Transportation Research Part B: Methodological, vol. 77, pp. 123-145, 2015.

[28] S. Timotheou, C. G. Panayiotou, and M. M. Polycarpou, "Distributed traffic signal control using the cell transmission model via the alternating direction method of multipliers," IEEE Transactions on Intelligent Transportation Systems, vol. 16, no. 2, pp. 919-933, 2015.

[29] N. R. Council, Highway Capacity Manual, Transportation Research Board, Washington, DC, USA, 2000. 


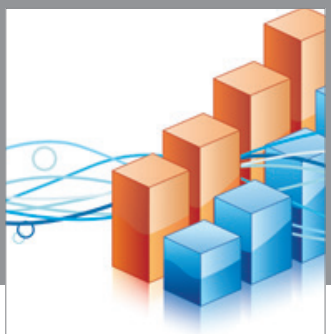

Advances in

Operations Research

vatem alat4

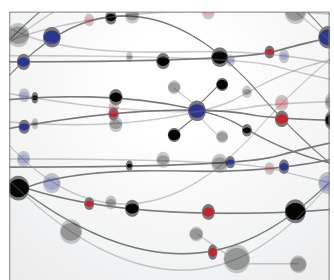

\section{The Scientific} World Journal
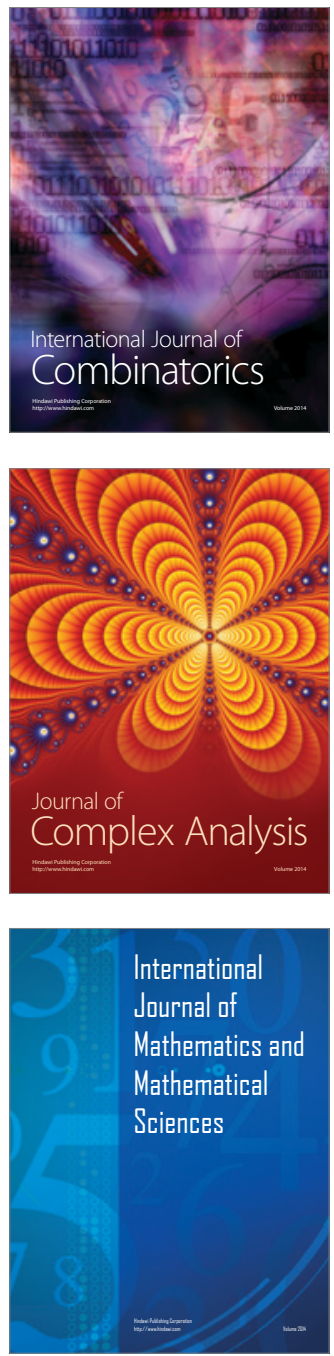


Algebra

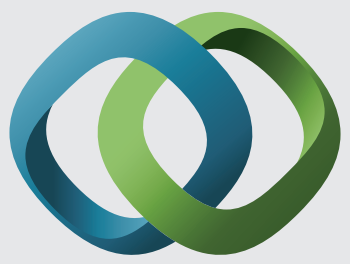

\section{Hindawi}

Submit your manuscripts at

http://www.hindawi.com
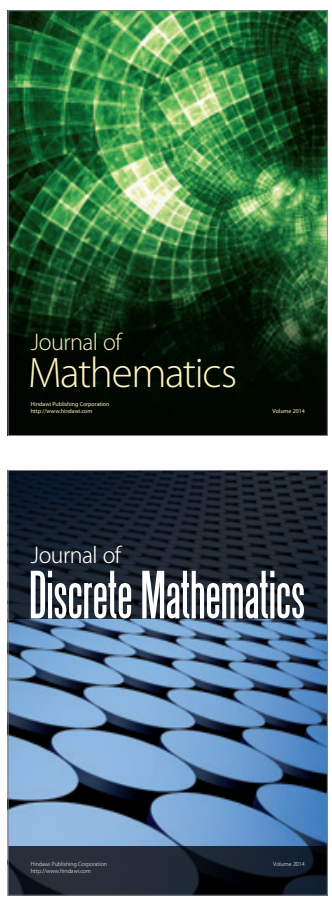

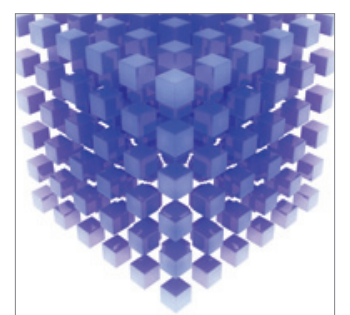

Mathematical Problems in Engineering
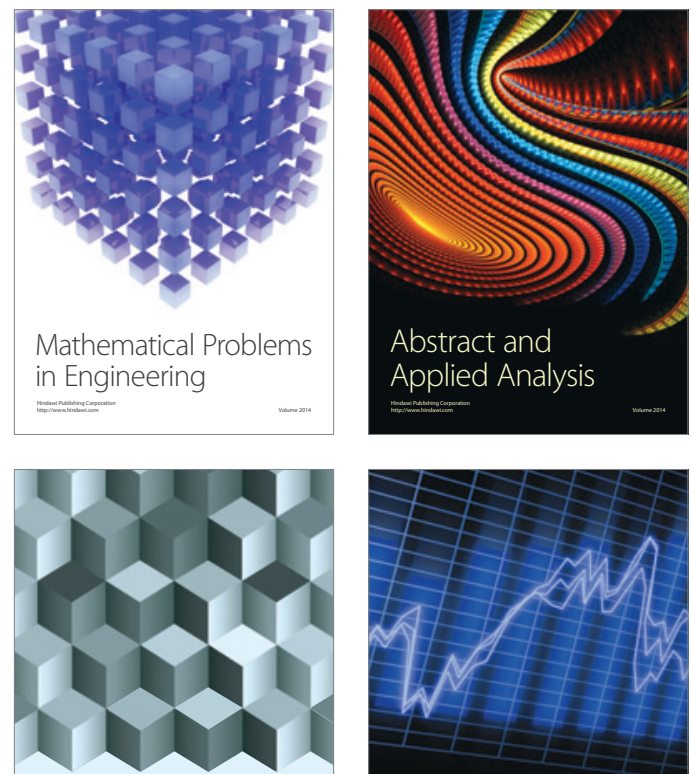

Journal of

Function Spaces

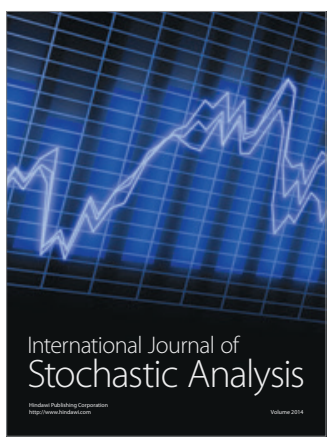

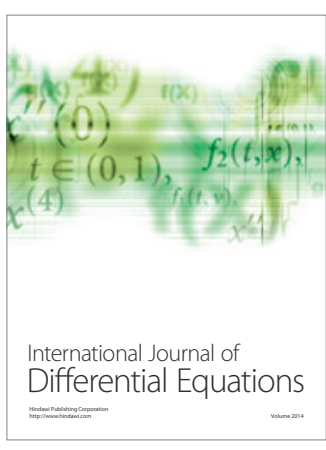
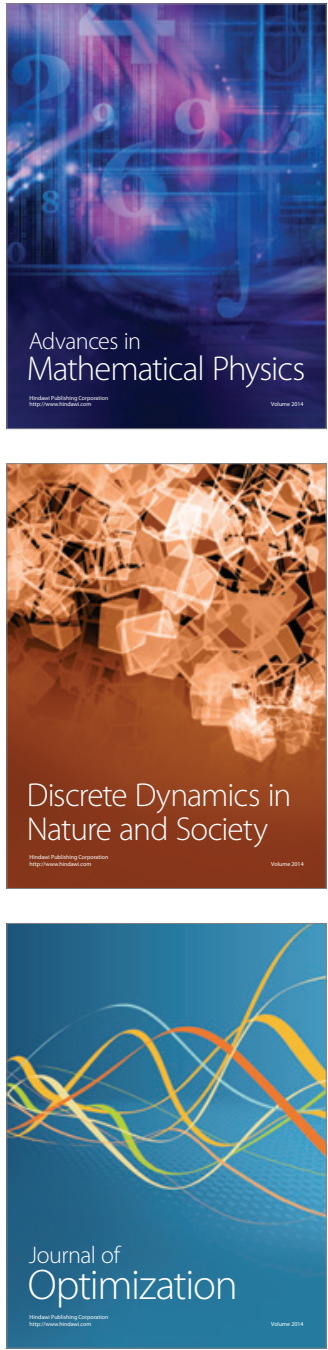